\title{
Martha C. Nussbaum - Otro Enfoque para la Defensa del Ser Humano y de los Derechos de las Mujeres
}

\section{Martha C. Nussbaum - Another Approach for the Defense of the Human Being and the Human Rights of Women}

\author{
Cristina Monereo Atienza \\ Universidad de Málaga, Málaga - Espanha
}

\begin{abstract}
Resumen: Este trabajo confronta las ventajas y desventajas de la teoría de las capacidades de Nussbaum en la búsqueda de la igualdad de las mujeres. Nussbaum entiende que los individuos en general y las mujeres en particular tienen una serie de capacidades comunes porque son fines en sí mismos. No se puede tratar a otra persona como un mero objeto, y esto merece un consenso transcultural sobre lo que es el ser humano. El concepto universal de sujeto que ofrece, basado en unos mínimos comunes a todos, abiertos al diálogo y el consenso político, es muy interesante. No obstante, no se pueden olvidar tampoco otros enfoques como el del discurso de los derechos.
\end{abstract}

Palabras clave: Nussbaum. Capacidades. Derechos de las Mujeres.

\begin{abstract}
This paper confronts the advantages and disadvantages of Nussbaum's theory in seeking equality for women. Nussbaum understands that people in general and women in particular have a number of common capabilities because they are ends in themselves. You can not treat another person as a mere object, and this deserves a cross-cultural consensus on what is the human being. The universal concept of the subject that she offers, based on a minimum common to all, open to dialogue and political consensus, is very interesting. However, we can not forget other approaches like the discourse of rights.
\end{abstract}

Keywords: Nussbaum. Capabilities. Rights of Women.

Recebido em: 02/03/2015

Revisado em: 20/04/2014

Aprovado em: 16/05/2015 


\section{Introducción: Martha C. Nussbaum y la Teoría de las Capaci- dades}

Martha Craven Nussbaum es una filósofa que comenzó a interesarse por la tragedia griega así como por buscar una relación más estrecha entre filosofía y literatura. Sus primeros trabajos conceden una gran importancia a la imaginación frente al valor tradicional de la razón, y buscan un determinado valor ético a las emociones para corregir el intelectualismo racionalista. (AGRA ROMERO, 2001, p. 336)

Estos inicios le conducirían posteriormente a interesarse por la teoría de las capacidades inicialmente abordada por el economista Amartya Sen. Sen considera que la perspectiva económica tradicional es deficiente en la comprensión de diversas cuestiones filosófico-políticas y socio-económicas, como la calidad y el nivel de vida, el bien-estar (well-being que no welfare), el desarrollo humano y las evaluaciones sociales e individuales sobre la libertad y la igualdad. Por ello, decide ampliar el conocimiento de estas materias a través de un concepto sobre la libertad para ser o hacer al que llama "capacidad" (capability). Las capacidades designan las combinaciones alternativas que un individuo puede hacer o ser, es decir, los distintos funcionamientos que puede lograr (SEN, 1999, 2002). Nussbaum supo considerar las ventajas de esta teoría, y durante la década de los ochenta comenzó a colaborar con Sen en temas relacionados con el desarrollo y la ética.

El enfoque de las capacidades (capabilities approach) es principalmente liberal ya que se relaciona directamente con el término de autonomía ética de Kant. Pero como bien haría notar Nussbaum, al mismo tiempo se conecta con las nociones de función o érgon de Aristóteles y con el concepto de necesidades de Marx (MONEREO ATIENZA, 2012, p. 53 y ss.). Nussbaum no solamente va a confirmar estas conexiones, sino que además va a dotar a la teoría de las capacidades de unas aportaciones personales muy relevantes que inciden sobre elementos tan cardinales como las cuestiones distributivas, el criticismo racionalista y universalista de raíz aristotélica, la lucha contra posiciones relativistas, y otros aspectos que enriquecen la teoría desde la perspectiva de género. 
En definitiva, Nussbaum está más cercana al perfeccionismo, y eso le hará enfatizar la relación entre capacidades y florecimiento humano, que es fundamental en la defensa del ser humano y sus derechos (GASPER, 1997, p. 281; MONEREO ATIENZA, 2010, passim). Hasta tal punto es esencial su visión que, actualmente, el enfoque de las capacidades se considera perteneciente a ambos autores. (SANCHEZ GARRIDO, 2008, p. 163)

\section{La Defensa del Ser Humano Desde la Teoría de las Capacidades}

La teoría de Nussbaum parte de la defensa de un "ser humano" con capacidades y necesidades comunes a todos independientemente del género, la clase, la raza o la nacionalidad (NUSSBAUM, 1995, p. 61; 2012). Piensa que este punto de partida es fundamental para la igualdad y la dignidad de las mujeres. Todos los individuos en general y las mujeres en particular son, como diría Kant, fines en sí mismos y no instrumentos para otros (no son meras reproductoras, encargadas de los cuidados familiares, ni objetos de descarga sexual. (NUSSBAUM, 2002, p. 28)

Esta perspectiva se centra en lo que todos los humanos comparten, antes que en los privilegios o logros del grupo dominante. Se basa en necesidades y funciones básicas de todos, antes que en poderes y estatus. (NUSSBAUM, 1995, p. 62)

Esta concepción está íntimamente ligada con la noción aristotélico- marxiana de dignidad humana, una noción que atraviesa las fronteras culturales (NUSSBAUM, 2002, p. 114). No se puede tratar a otra persona como un mero objeto, y esto merece un consenso transcultural porque el ser humano es una criatura necesitada de una pluralidad de actividades vitales. La racionalidad es un aspecto de ese animal, pero existen también otros de vital importancia. Esta visión acepta la dignidad de todos, incluyendo la de aquellas personas discapacitadas cuya razón está mermada. (NUSSBAUM, 2002, p. 167)

El hecho de buscar un concepto universal, esencialista, del ser humano ha recibido muchas críticas por parte de ciertos grupos que defienden la incidencia de las diferentes culturas en los seres humanos y, 
también, por parte de cierto femenismo que sigue manteniendo actitudes escépticas ante enfoques universales del sujeto. (CHARLESWORTH, 2000, p. 64 ss.)

Efectivamente, el androcentrismo del sujeto moderno ha sido una constante en el pensamiento. Para autores como Inmanuel Kant, John Locke o Jean Jacques Rousseau la mujer era por naturaleza distinta e inferior al hombre (PATEMAN, 1988, p. 172). Kant no incluye a las mujeres en el sujeto universal ni en el contrato. Las mujeres no son sujetos éticos ni políticos. No están incluidas en la ciudadanía (AMORÓS, 2008, p. 26). En todo caso, son simples "objetos" del contrato matrimonial donde se impone el derecho del hombre a la posesión de la mujer (PATEMAN, 1988, p. 172). También Locke (1824, p. 38) al hablar de la sociedad familiar opina que la mujer debe obediencia al marido "al que pertenece". Rousseau $(1820 ; 1855)$ está de acuerdo en que las mujeres están confinadas en la esfera privada, y sus obligaciones son las de esposa y madre, mientras los hombres están preparados para ser ciudadanos en una democracia participativa, y para expresar sus pensamientos y deseo libremente. Más tarde, el irracionalismo romántico rechaza el periodo anterior y a la razón liberal, pero no renuncia al uso de la naturaleza para definir a la mujer (PULEO, 1992, p. 13). Las mujeres son "esencias" por naturaleza y, en última instancia, lo que los varones piensan sobre esa naturaleza. Para Arthuer Schopenhauer (1961, p. 376) las mujeres no son sino imitación y puro deseo de agradar a los hombres. Para Soren Kierkegaard (2006, p. 137), la mujer no "existe” por sí misma e, incluso, el verbo "existir" es demasiado para ella. Del mismo modo, el falocentrismo de Sigmund Freud han recibido abundantes críticas.

Ante este uso "intencionado" de la naturaleza humana, es lógico que se vea con cierto escepticismo cualquier definición universal del sujeto. No es de extrañar tampoco que el feminismo haya intentado distanciarse de las propuestas modernas. Desde Simone de Beauvoir aparece una nueva forma de reflexión sobre la "cuestión de las mujeres". Beauvoir no quiere caer en esencialismos, por eso toma una actitud existencialista en la que la mujer no es nada concretado por naturaleza. Se considera un ser que "se hace". Desde su punto de vista no se pueden hacer 
comparaciones entre hombres y mujeres ni entre sujetos porque todos son diferentes. (BEAUVOIR, 2002, p. 428)

Sin profundizar en la teoría de Beauvoir, es cierto que la "diferencia” empezó a ser la reivindicación por excelencia del feminismo. Para algunas llevó a la configuración de una identidad autodesignada apelando a la antinomia de sexos (IRIGARAY, 2007, p. 119 y ss.). Otro tipo de feminismo luchó por incluir los temas de raza y cultura en la perspectiva de género. (HOOKS, 1984, p. 25)

No obstante, muchos de estos discursos tienen inconvenientes al predicar el postmodernismo y la muerte del sujeto. No hay un sujeto alternativo con mecanismos para la emancipación humana. A pesar de las críticas postmodernas, parece que la razón subjetiva es la única fuente de normatividad. Por eso, es preciso reconstruir la noción del sujeto atacando el androcentrismo reinante. (AMORÓS, 2008, p. 358 y ss.)

Del mismo modo ocurre con las críticas al universalismo de los defensores del comunistarismo cultural. La crítica comunistarista se preocupa del individuo como ser social e íntimamente relacionado con su entorno cultural y ambiental. Charles Taylor (1989, p. 26) acusa al liberalismo por considerar atomísticamente a los individuos y por pensarlos como seres independientes y autónomos de su propio contexto. Desde su visión, no se puede concebir un sujeto universal con características comunes porque cada individuo es necesariamente diferente según su entorno. Con todo, la tendencia comunistarista tiene el peligro de disolver al individuo en el grupo identitario cultural.

La teoría de las capacidades de Nussbaum lanza una propuesta para conseguir corregir los defectos de la razón y el sujeto ilustrado y, además, considerar la diversidad de los individuos. Nussbaum elabora un concepto universal de sujeto, admitiendo unos mínimos comunes a todos los individuos independientemente del sexo. De esta forma se recuperan algunas propuestas históricas como la de John Stuart Mill y la de Karl Marx. Para Mill $(1991$, p. 153, 211) la naturaleza de la mujer es un producto artificial que ha conducido a aptitudes propicias a los intereses y placeres de los varones. Pero el ser humano es universal y lo definen unas características básicas sin las cuáles sería "menos persona". Del mismo modo la idea de 
dignidad humana y necesidades básicas de Marx (2004) apelan a un concepto humano común.

Nussbaum (1995, p. 63; 2002, p. 32, 34) no tiene ningún problema en aceptar que su propuesta es "universal" y "esencialista no metafísica". Prefiere centrarse en los aspectos comunes aunque al mismo tiempo respetando las diferencias que dividen. Esta idea difiere de Sen. Sen es crítico con la noción de sujeto moderno egoísta y aislado, y propone un sujeto situado en su entorno. Sin embargo, no se atreve a fijar unos mínimos que describan al ser humano en general. De sus teorías se desprende el intento de hablar de una "humanidad compartida", aunque no fija ningún elenco de funcionamientos ni capacidades que la puedan definir. Sen es temeroso de las nociones esencialistas y está más próximo al nominalismo moderado según el cual ser humano se construye continuamente aunque pueda hablarse de algunos constituyentes básicos comunes. Por su parte Nussbaum va más allá. Su posición es realista (no metafísica) y se atreve a fijar una lista de funcionamientos y capacidades sin las cuales un ser no es considerado "ser humano". Para Nussbaum fijar esa lista es importante para evitar el relativismo cultural que tanto ha acechado a Sen (GASPER, 1995, p. 293 y ss.). Ahora bien, su propuesta no es metafísica porque no parte de ninguna naturaleza inmutable. Muy al contrario, su lista le parece un punto de partida muy aceptable para comenzar un diálogo abierto y autocrítico (NUSSBAUM, 1995, p. 70). Si las capacidades eran para Sen un espacio de evaluación, para Nussbaum (2002, p. 112) son principios políticos básicos sucritos como garantías constitucionales exigibles. Así, el compromiso de los gobiernos sería el de asegurar las bases sociales para el desarrollo de las mismas. Su propuesta es una teoría más bien parcial o incompleta sobre la justa distribución, porque es compatible con diferentes visiones de la distribución por encima del mínimo fijado (NUSSBAUM, 2007, p. 87, 274). La iniciativa no excluye otras maneras de entender el ser humano, ni es imperialista porque no imponen un plan de vida único ni tampoco conduce a la uniformidad. Nussbaum es buena conocedora de la historia y los mitos antiguos y esto le ha servido como punto de referencia para examinar la gran variedad de interpretaciones sobre los seres humanos en muchos momentos y lugares. La conclusión a la que ha llegado es que la gente de sociedades diferentes compar- 
ten una visión general muy parecida. Esto no es sorprendente cuando, en realidad, todos los seres humanos se reconocen como miembros de una misma especie, se unen entre ellos, tienen descendencia, etc...La lista de capacidades de Nussbaum no es ahistórica ni a priori, sino que se basa en experiencias compartidas a lo largo de la historia. Su pretensión es reconocer la humanidad más allá de las fronteras.

Es una concepción normativa y valorativa y, por tanto, abierta al diálogo y el consenso político. Hay que insistir en que no es una teoría instrumental de la justicia sino orientada al resultado (NUSSBAUM, 2002 , p. 98). Identifica una lista de capacidades humanas, esto es, "aquello que la gente es realmente capaz de hacer o ser, de acuerdo a una idea intuitiva de la vida que corresponda a la dignidad del ser humano" y las coloca en el contexto de un tipo de liberalismo político que las convierte en metas políticas. Las capacidades carecen, por tanto, de toda fundamentación metafísica.

Nussbaum está cerca del perfeccionismo de Aristóteles pero, a la vez, del liberalismo de Kant. Nussbaum (2007, p. 29 y ss.) es muy crítica con algunos aspectos de las teoría contractualistas como el estado de naturaleza, pero confía en que los seres humanos tienen experiencias y concepciones solapadas, y que el consenso en este sentido es posible dentro de procedimientos razonables. Para algunos la teoría de las capacidades no está cerca del contractualismo kantiano, porque en el diálogo solamente se requiere fe en la democracia y en que se discutirán los temas conforme a unos principios sin que el resultado tenga que ser correcto ni razonable (PUTNAM, 2008, p. 377-388). Pero Nussbaum afirma que la noción de racionabilidad es esencial, aunque múltiple, y tiene además un contenido normativo.

Por lo demás, la lista es heterogénea y su inicio está en la idea intuitiva del ser como ser capaz y necesitado al mismo tiempo (NUSSBAUM, 2002, p. 119). John Rawls ha criticado el instuicionismo para derivar principios de justicia, pero Nussbaum piensa que tanto la teoría de Rawls como la suya se basan en juicio ponderados y consistentes pero en fases distintas. En el caso de Rawls hay juicios de ponderación en la posición original (aunque no luego en la determinación de una lista de bienes) y, 
asimismo, juicios de ponderación en la elaboración de una lista de capacidades mínimas (NUSSBAUM, 2007, p.179 y ss.). En el caso de Nussbaum, el intuicionismo es perceptible a la hora de realizar el elenco de capacidades humanas. De hecho, su concepto de ser humano tiene que ver con el concepto normativo ético moderno de "persona", pero justamente ha evitado acuñar este término para evitar falsas neutralidades y tremendas exclusiones (como la de las mujeres, por ejemplo). (MOLLER OKIN, 1995, p. 280)

\section{La Lista de Capacidades Humanas}

A partir de las líneas hasta ahora expuestas, Nussbaum traza distintos niveles sobre la concepción del ser humano, dentro siempre de una lista sobre la que trabajar y discutir. El primer nivel concibe la forma de vida humana. Realiza un índice con aquellas cosas comunes a cualquier vida humana. En primer lugar, la mortalidad. Afirma Nussbaum que todos los seres humanos se enfrentan a la muerte y sienten cierta aversión hacía ella. En segundo lugar, el cuerpo humano. Todo ser humano tiene determinadas necesidades y capacidades, aunque la forma de vivirlas y desarrollarlas sea un hecho cultural. Entre ellas, la necesidad de comida y bebida, necesidad de abrigo, deseo sexual y movilidad. En tercer término, todo ser humano tiene capacidad para el placer y el dolor. En cuarto lugar, la capacidad cognitiva (para percibir, imaginar y pensar). En quinto lugar, está necesidad de desarrollo desde la primera infancia. En sexto punto, es común la razón práctica. Todo ser humano participa o intenta participar en la planificación y dirección de su propia vida preguntándose sobre lo que es mejor. En séptimo lugar, la asociación con otros seres humanos. Ya lo decía Aristóteles, el hombre es un ser sociable. En octavo lugar, la identificación de que se es miembro de una especie que convive con otras especies animales distintas. En noveno puesto, Nussbaum habla del humor y el juego, esto es, de la risa y el ocio. En décimo término, el sentimiento de diferenciación individual. El ser humano es capaz de distinguir que es uno y que es él solo el que siente dolor o placer, por ejemplo. Por último, cada ser humano es consciente de su entorno y contexto que es diferente al de otros. 
Esta enumeración, que se ha traslado brevemente aquí, indica lo más básico del ser humano de tal manera que, como decía Mill, sin estas cualidades un ser humano es "menos persona", no es un ser humano en absoluto y, por tanto, tampoco se puede considerar esa vida como una vida humana "buena". Nussbaum (1995, p. 80) está determinando una concepción mínima del bien en la que no se estipula un modo de vida pero sí unos parámetros de elección de esa vida. No se ofrece el bien en sí, sino la base social del bien.

El problema es ahora fijar los límites de estas capacidades básicas (NUSSBAUM, 2007, p. 83). Por una parte, está ese umbral en el que la capacidad es tan pobre que no se puede considerar humana. Por otro, el umbral tiene que ver con que estas capacidades y funciones son tan reducidas que nadie podría considerar esta forma de vida como una "vida humana buena". En el primer umbral se puede evaluar que alguien mal nutrido puede vivir pero que esto no se puede considerar una vida digna. El segundo umbral se refiere a las capacidades mínimas que se consideran desde el punto de vista de la justicia de un país o comunidad. El objetivo de las políticas públicas son, así, las capacidades básicas (no ya las funciones o funcionamientos). Por ejemplo, una persona puede elegir una vida célibe aunque contenga la capacidad de expresión sexual. Así se evita caer en paternalismos que limiten las opciones. Con todo, es cierto que algunas políticas públicas son más eficaces si se centran en los funcionamientos directamente. Al fin y al cabo, cierto parternalismo es inevitable para defender la libertad porque existen también otro bienes jurídicamente protegibles que hay que incentivar, como por ejemplo el medio ambiente o la salud pública (DENEULIN, 2002, p. 502-506). Nussbaum acepta que la asistencia en momentos de dependencia aguda forma parte de las necesidades primarias de los ciudadanos cuya satisfacción constituye una rasgo esencial de una sociedad justa. Sin embargo, la asistencia no debe entrar en ninguna lista de manera individualizada. Al contrario, se ha de remitir a todas las capacidades en general. (NUSSBAUM, 2007, p.174-175)

De este modo, se llega al segundo nivel relacionado con las capacidades humanas básicas funcionales. Nussbaum (1995, p. 83 y ss.) también las enumera: ser capaz de vivir el tiempo normal de una vida huma- 
na; se capaz de tener buena salud (estar bien nutrido, tener abrigo, tener oportunidades para la satisfacción sexual y reproducción, poder moverse de un sitio a otro); ser capaz de evitar dolor innecesario y nada beneficioso y tener experiencias placenteras; ser capaz de usar los sentidos (de imaginar, pensar y razonar y, por tanto, de ser educado); ser capaz de tener sujeción a cosas y personas; ser capaz de formular la propia concepción del bien y comprometerse con la planificación de la propia vida (trabajar fuera de casa y participar en la vida política); ser capaz de vivir por y para los demás; ser capaz de vivir en relación y respeto a los demás animales, plantas y el resto de la naturaleza; ser capaz de reír, jugar y divertirse; ser capaz de vivir la propia vida sin interferencias en las elecciones individuales; ser capaz de vivir la propia vida en el propio contexto (lo que conlleva la garantía de ciertas libertades de asociación y propiedad, y límites a las mismas).

Según afirma Nussbaum, no cabe satisfacer una de estas necesidades dando mayor cantidad de otra. Las capacidades no son intercambiables ni negociables. Todas son de importancia capital, aunque destacan la razón práctica y la afiliación. El hecho de planificar la propia vida necesita de la razón práctica y también del concurso de los demás. Por ejemplo, el trabajo para ser un modo de funcionamiento verdaderamente humano, ha de implicar la razón práctica y la afiliación: ha de implicar la capacidad de que uno se comporte como ser pensante y de poder hacerlo junto a otros. Al destacar estos dos puntos, Nussbaum no quiere decir que la salud, por ejemplo, sea un mero medio para la libertad de elección. Pero sí está afirmando que no es suficiente que el gobierno promueva el acceso a la salud en modo reducido. La meta de una planificación de las políticas públicas debe ser garantizar suficientemente conforme a la a la razón y la afiliación las capacidades de todos los ciudadanos para realizar funciones importantes y valiosas. Solamente así puede medirse la calidad de vida de un país o comunidad. Como para Sen, las capacidades son una demanda moral, no necesariamente (aunque sí preferiblemente) legalizada, que puede identificarse con una serie de derechos fundamentales (esta vez, de todo tipo), los cuales deben garantizarse en cualquier sociedad. Los derechos de primera y de segunda generación se entralazan inseparablemente. 
Nussbaum (2007, p. 128-129) distingue las capacidades básicas como equipamiento innato de los individuos, las capacidades internas identificadas con estados desarrollados de la persona misma que son condición suficiente para el ejercicio de la función, y capacidades combinadas que son capacidades internas ordenadas con adecuadas condiciones externas para el ejercicio de la función. Obviamente, entre todas estas capacidades existe una línea muy estrecha de unión. Así, una distinción entre capacidades internas y combinadas puede verse en el caso de las mujeres que quiere trabajar fuera del hogar y tienen habilidades para hacerlo, pero se ven impedidas por razones familiares o religiosas. El enfoque de las capacidades, por tanto, es fundamental para medir la igualdad y la calidad de vida de las mujeres. De todos modos, Nussbaum (2007, p. 319 y ss.) entiende que en relación a las mujeres se debería tener en cuenta además y de manera más profunda la institución familiar, no como una institución natural sino como una organización social en la que todos sus miembros son fines en sí mismos.

\section{Por Qué la Teoría de las Capacidades para la Defensa del Ser Humano en General y los Derechos de las Mujeres en Particular}

El enfoque de las capacidades puede triunfar donde otros enfoques han fracasado. Nussbaum, como Sen, critica el enfoque utilitarista porque solamente tiene en cuenta a las personas en función de la satisfacción de su preferencias. Esta perspectiva ignora que los deseos y preferencias subjetivas no siempre son indicadores fiables de lo que realmente necesitan las personas. Para Nussbaum (1995, p. 92), dar prioridad a las preferencias de una cultura tradicional puede ser especialmente subversivo para la calidad de vida de las mujeres. Tampoco acepta, como Sen, el enfoque liberal que busca la equidad en la distribución de ciertos recursos básicos o bienes materiales. Como Sen, afirma que la riqueza y los ingresos no son buenos en sí, sino en cuanto son medios para promover las funciones humanas. La teoría de los bienes materiales como la de Rawls cosifica las relaciones humanas. Para algunos, tomar algo objetivo como medida de la desigualdad es preciso, porque el resto de propuestas se refieren a aspectos intangibles que bien pueden ser interpretados de uno u otro 
modo (GLOVER, 1995, p. 121). Incluso, la misma capacidad de razonamiento de la teoría de Nussbaum puede ser matizada hasta el punto de distinguir entre mujeres y hombres en base a una menor racionalidad y un mayor sentimiento en el sexo femenino (no es, desde luego, lo que pretende Nussbaum -2008- al matizar la importancia de la razón y valorar las emociones en todos los seres humanos en general). Con todo, no parece que a nivel básico las capacidades puedan diferir hasta el punto de dividir radicalmente entre hombres y mujeres. Además, los seres humanos siempre van a tener distintas necesidades materiales y distintos impedimentos según sus circunstancias, y esto ha de ser medido de igual manera. El criterio de los bienes materiales no es tan objetivo como pretende ser.

La teoría de la capacidades de Nussbaum contempla las circunstancias históricas y las diferencias culturales ya que la lista es muy general y perfectamente constitutiva de cualquier vida humana que se desarrolla diversamente. Las diferencias en este sentido no son radicales, siempre hay solapamientos y puntos para el debate y la autocrítica. Nussbaum rechaza el ataque liberal según el cual tal lista no respeta la autonomía de los individuos para tomar sus propias decisiones sobre una buena vida. Los funcionamientos y las capacidades, aunque relacionadas, son distintas. Las funciones informen de las capacidades, pero no son las capacidades mismas. No se impone, por tanto, ningún modelo de vida buena que tengan que alcanzar necesariamente los individuos. El enfoque de Nussbaum ofrece oportunidades de acción pero no decide directamente. La capacidad más importante es, precisamente, la capacidad de decidir. Esto evita cualquier tipo de paternalismo que imponga una determinada concepción de la vida. En este sentido, la filósofa se acerca a Kant y, al mismo tiempo, a Aristóteles puesto que no ofrece un concepto de vida buena, pero sí indica con su lista un concepto grueso del bien que determina los fines para una vida buena y límites y fronteras para un diálogo basado en la razón (O’NEILL, 1995, p.145 y ss.; BENHABIB, 1995, p. 254), y vago a la vez porque no especifica esos fines (ARNESON, 2000, p. 49). Es una teoría que valora los deberes pero que insiste en que no puede construirse una teoría de la justicia sobre los deberes (O'NEILL, 1996) porque los derechos son prioritarios. (NUSSBAUM, 2007, p. 275) 
En definitiva, las mujeres como seres humanos tienen la capacidad de decidir sobre su propio concepto de vida buena. Se trata de una cuestión de justicia. El problema es que no siempre se ha considerado a la mujer un "ser humano" con las mismas características que el hombre. Se ha dicho que mujeres y hombres tienen naturalezas distintas. Pues bien, no parece razonable defender una naturaleza diferente de ambos sexos como tampoco parece razonable argumentar que los sexos cumplen roles sociales divergentes y que, por tanto, deben desarrollar tales capacidades en esferas distintas. Ni siquiera ha de ocurrir en sociedades culturalmente distintas, al menos en el nivel básico que plantea la teoría de las capacidades.

Por un lado, como se sabe, la naturaleza es un concepto maleable que ha sido definido intencionadamente de una u otra manera para beneficiar al grupo de individuos que detentan el poder. El mismo concepto de "sujeto" ha estado referido al varón blanco, heterosexual, occidental y de clase medida. Por otro, se ha querido definir la naturaleza en base a la biología, y de este "hecho" se han querido deducir unos roles sociales que determinan lo que los individuos "deben hacer o ser" en una sociedad. Sin embargo, es importante distinguir entre las nociones de "sexo" y "género". Mill $(1991$, p. 164, 198) separaba a mujeres y hombres no en función de las diferencias sexuales sino en función de la organización de la sociedad, la educación y la familia. No cree que pueda distinguirse entre mujeres y hombres por razones biológicas. Más bien, ha sido la sociedad la que artificialmente ha dividido los sexos estableciendo una posición y unos roles sociales concretos. Para muchos autores, la biológica afecta a la personalidad de los individuos y, por eso también, hombres y mujeres tienen roles sociales distintos. Como se explicaba anteriormente, Mill (1991, p. 211) defiende que la ciencia no dice nada acerca de la verdadera naturaleza de los seres humanos y de las influencias sociales que hayan podido determinar su carácter. La naturaleza de la mujer es un producto artificial construido para satisfacer los intereses de los hombres. Asimismo, para Beauvoir el individuo no es nada en esencia y se va haciendo en potencia (AMORÓS, 2001, p.106). Beauvoir distingue también entre sexo (naturaleza) y género (cultura) (aunque es cierto que actualmente este enfrentamiento entre sexo y género es discutible) (BUTLER, 1990, passim). a mujer es naturaleza igual que lo es el hombre, pero es, 
sobre todo, género construido en desigual jerarquía social y roles. Beauvoir critica la opresión histórica que ha sufrido socialmente la mujer en el sistema patriarcal, y anima a las mujeres para encontrar modelos alternativos a la vida de esposa y madre. Frente a lo que pensaban algunos como Freud, en el mundo actual las diferencias biológicas no determinan el desarrollo humano (BEAUVOIR, 2002, p. 58). Lo relevante son los valores derivados de los proyectos personales. Así pues, lo que ha de tenerse en cuenta no es el "sexo" sino la elección libre de proyectos humanos. (BEAUVOIR, 2002, p. 113)

Recordar de nuevo estos ejemplos de grandes pensadores como Mill o Beauvoir confirma la necesidad de formular un concepto universal de ser humano que no distinga entre mujeres y hombres a priori ni caiga en dogmatismos y formulaciones discriminatorias. Las distinciones biológicas entre mujeres y hombres no son relevantes al nivel básico planteado por la teoría de las capacidades. Es cierto que centrarse en algunos aspectos de la biología humana para hacer una lista es una empresa peligrosa (WOLF, 1995, p. 106) pero, aunque pudieran existir diferencias de sexo, no son tan trascendentes como para perturbar las capacidades de elección. En cambio, las diferencias de género sociales sí son apreciables en cuanto pueden afectar a la toma libre de decisiones sobre la propia vida. Por eso, habrá que actuar positivamente sobre estas diferencias y discriminaciones construidas artificialmente si se quiere conseguir la auténtica igualdad entre mujeres y hombres.

Además, separar en una lista lo que se entiende por una vida humana (objetivamente) de lo que se considera una vida humana buena (subjetivamente) es una propuesta fuerte de Nussbaum para enfrentarse directamente al relativismo cultural sin caer en el elitismo (ARNESON, 2000, p. 50). Ciertamente, la primera aproximación a un concepto del bien puede irradiar un irrefutable elitismo al estilo nietzscheano según el cual solamente las metas más altas de los mejores especimenes de la humanidad tienen algún valor. No obstante, esta idea es discutible. El hecho de que, por ejemplo, no se tengan tales capacidades en caso de discapacidad no hace "caer" la validez de la lista ni la hace "elitista", porque, en realidad, a todo el mundo le gustaría poder tener esas capacidades, es decir, que son valiosas para todos. Nussbaum dedica especial atención al tema de la 
discapacidad. Sabe que las teorías modernas constractualistas de justicia han ignorado el tema de la discapacidad y piensa que el enfoque de las capacidades está mejor preparado para responder ante estas cuestiones en cuanto parte de una concepción de persona como animal social cuya dignidad no depende de una racionalidad idealizada sino también de otros muchos factores vitales.

Por otra parte, se ha dicho que Nussbaum debería haber tenido en cuenta que dentro de las sociedad actuales, tal y como están organizadas política y socio-económicamente, no se pueden potenciar todas estas capacidades para todos los individuos a la vez y se tiene que reflexionar sobre quien debe ser beneficiado primero (WOLF, 1995, p. 111). No se sabe si, dado el caso, habría que ayudar, por un lado, a aquellos que están más lejanos de cumplir con esas capacidades para mejorar su situación sin que, no obstante, se rebase el umbral marcado, o, por otro lado, ayudar a los que aún estando necesitados están cercanos a la línea socialmente establecida y con esa ayuda pueden pasar el umbral. Nussbaum parece estar de acuerdo en ayudar a los que están cercanos al umbral y no a los que están peor situados (aunque estos sean más). El perfeccionismo supone la maximización de la excelencia humana (ARNESON, 1999, p. 179). Este principio se enfrenta al criterio de prioridad de los peor situados (aunque sin tener que llegar al "lexism" (ARNESON, 2000, p. 59). Lo ideal, sin duda, es pensar que se trata de un nivel muy básico y, ciertamente, todo estado debería promover estas capacidades para todos sin excepción. Esto significaría optar por una restructuración económico-social de todo el sistema, opción que puede sonar utópica (en el sentido despectivo). Aún con todo, la propuesta de las capacidades es la meta deseable y no vale hablar, por ejemplo, de escasez de recursos, sino desgraciadamente de modos de producción y distribución de bienes inadecuados. (MONEREO ATIENZA, 2005, p. 273 y ss.; 2007)

En suma, la propuesta de Nussbaum puede ser vista como un índice de derechos cuya fundamentación está en el consenso solapado entre diferentes culturales y cuyos rasgos básicos son: la razonabilidad múltiple en base a preferencias individuales y circunstancias locales y tradicionales; la capacidad como meta y no los funcionamientos los cuales quedarían a elección de los individuos (agencia y no bienestar); las libertades civiles 
y políticas y la razón práctica en el deliberación; el liberalismo político y el consenso superpuesto entre personas con visiones comprehensivas muy diferentes acerca del bien; y las restricciones en la implementación de los principios derivados de la lista. Todo esto es muy positivo desde la perspectiva de género.

Además, Nussbaum (2007, p. 184) sostiene que todas la capacidades son igualmente fundamentales. No prima directamente la libertad como lo hace Sen, pero está de acuerdo en que en ningún caso se pueden imponer las necesidades económicas negando la libertad. Este era uno de los puntos criticables de la perspectiva de Sen, quien parece primar los derechos individuales, civiles y políticos sobre los derechos sociales y culturales. Para Nussbaum todos los derechos (individuales civiles y políticos, por un lado, y económicos, sociales y culturales, por otro) tienen igual importancia, aunque puedan diferir en algunos aspectos. Esto tiene que ver con el tema de las capacidades combinadas: sólo se tiene la capacidad de ejercitar un derecho si existen las condiciones adecuadas para hacerlo. La libertad real está muy relacionada con la igualdad.

El lenguaje de los derechos sigue teniendo un gran valor pero tiene varios defectos que pueden ser subsanados con el enfoque de las capacidades: el defecto de estar asociados a la cultura occidental, el defecto de primar los derechos de primera generación sobre los derechos de segunda generación, y el defecto de implicar históricamente una separación entre la esfera pública y la esfera privada. Así, en primer lugar, las capacidades pueden ser un término universal común a todas los individuos y culturas (NUSSBAUM, 2007, p. 147). En segundo lugar, esta terminología ayuda a crear una nueva fórmula de interdependencia de los derechos. Por una parte, los derechos tienen tanto una dimensión negativa como positiva ( $\mathrm{p}$. 285). Por otra, todos los derechos están interrelacionados (p. 288). El enfoque de las capacidades insiste en los aspectos materiales de los bienes humanos al dirigir la atención a lo que las personas son capaces de ser o hacer. En tercer lugar, las capacidades ayudan a compensar las desigualdades que sufren las mujeres dentro de la familia (desigualdades en los recursos y las oportunidades, déficit educativos, reconocimiento del valor de su trabajo de asistencia, violaciones a su integridad corporal). Esto puede ser un punto de partida para la reformulación del espacio público. 
De todos modos, Nussbaum sigue usando el valor de la libertad para fundamentar todos los derechos y define la igualdad, sobre todo, como igualdad en la libertad. Esto quiere decir que, como Rawls, los derechos individuales, civiles y políticos se aplican bajo el criterio estricto de la igualdad mientras los derechos económicos, sociales y culturales tienen que adecuarse a la suficiencia de recursos de las circunstancias político-jurídicas y socio-económicas. Esto es pragmático al día de hoy pero es criticable.

Todos los derechos individuales y sociales van dirigidos a la satisfacción de una vida digna. La misma Nussbaum cree en la igual dignidad de todos los seres humanos. Esto puede ser discutido si se valora la igualdad solamente en la libertad y se observa secundariamente la igualdad en los resultados. Es reprochable porque al hablar de "suficiencia" de estos derechos frente a "igualdad" de las libertades se les deja dependientes del sistema socio-económico del contexto. Es, por supuesto, un avance e incluso es práctico defender la fijación de un mínimo básico suficiente e imprescindible para estos derechos (eso mismo se ha llevado a cabo ya con los derechos de primera generación), pero no se entiende teóricamente porqué ese mínimo tiene que regirse por la "suficiencia" y no la "igualdad". No parece que la razón sea la defensa de una concepción que entiende que los derechos sociales son derechos que generan obligaciones positivas mientras las libertades generan obligaciones meramente negativas. Se ha visto que las libertades tienen tanto un sentido negativo como positivo y, al fin y cabo, son necesarios también recursos para su despliegue. Quizás sea que los derechos sociales, efectivamente, necesitan más recursos materiales y se sigue primando como un hecho consagrado un determinado sistema socio-económico (el liberal-capitalista) que produce y distribuye los bienes de una manera concreta (MONEREO ATIENZA, 2004, p. 275).

\section{Conclusión}

A pesar de algunas críticas, hay que reconocer que la teoría de las capacidades de Sen y Nussbaum es una propuesta original para afrontar 
los temas de desigualdad social a partir del concepto de libertad. El énfasis en la libertad dota de una visión distinta del individuo necesitado como individuo potencialmente activo para elegir sus propios proyectos de vida.

Es un enfoque, en su conjunto, tampoco olvida las circunstancias materiales básicas para desarrollar las libertades y que tiene en cuenta, además, otras circunstancias no materiales que pueden afectar a su desarrollo.

En este sentido, es esencial para el feminismo luchar contra el sistema patriarcal y contra la discriminación histórica de género. Esto se debe hacer enfatizando la idea de un sujeto universal que no diferencie entre los sexos. Ha de promoverse, como lo incentivaba Beauvoir, el poder de las mujeres para decidir sus propios proyectos de vida. A la vez, es fundamental que los poderes públicos y todos los individuos actúen sobre las circunstancias sociales que organizan la sociedad separando la esfera pública y la esfera privada y estableciendo unos roles diferentes y distintamente evaluados para hombres y mujeres. Las mujeres parecen estar enclaustradas en la esfera privada sin posibilidades reales de salir de ella, y sufriendo faltas para el desarrollo de sus propios proyectos de vida. Es preciso reestructurar y reformular el sistema para erradicar esa visión enraizada que separa a dos sexos y margina a la mujer.

\section{Referencias}

AGRA ROMERO, M. X. Animales politicos: capacidades humanas y búsqueda del bien, de Martha C. Nussbaum. In: MAÍZ, R. (Comp.). Teorías políticas contemporáneas. Valencia: Tirant lo Blanch, 2001. p. 335-363.

AMORÓS, C. Simone de Beauvoir: un hito clave de una tradición. In: VALCÁRCEL, A.; ROMERO, R. (Ed.). Pensadoras del siglo XX. Sevilla: Instituto Andaluz de la Mujer, 2001. p. 103-122.

ARNESON, R. J. Human Flourishing versus Desire-Satisfaction. Social Philosophy and Policy, USA, v. 16, p. 113-142, 1999. 
BEAUVOIR, S. de. El segundo sexo. Traducción A Martorell. 6. ed. Madrid: Cátedra, 2002. (v. I y II)

BENHABIB, S. Cultural Complexity, Moral Interdependence, and the Global Dialogical Community. In: NUSSBAUM, M.; Glover, J. (Ed.). Women, culture and development. study of human capabilities. Oxford: Clarendon Press, 1995. p. 235-255.

BUTLER, J. Gender trouble - feminism and subversion of identity. London/New York: Routlege, 1990.

CAPABILITY and Well-being. In: NUSSBAUM, M.; SEN, A. (Ed.). The Quality of life. 6. reimpr. Oxford: Oxford University Press, 2002. p. 3053.

CREAR capacidades. Propuestas para el desarrollo humano. Traducción A. Santos Mosquera. Barcelona: Paídos, 2012.

CHARLESWORTH, H. Martha Nussbaum's Feminist Internationalism. Ethics, Chicago, v. 111, n. 1, oct., p. 235-255, 2000.

DENEULIN, S. Perfecionism, Paternalism and Liberalism in Sen and Nussbaum's Capabilities Approach. Review of Political Economy, USA, v. 14, n. 4, p. 497-518, 2002.

EMILIO. Madrid: Impr. del Semanario y de la Ilustración, 1855.

EMOTIONS and Women's Capabilities. In: NUSSBAUM, M.; GLOVER, J. (Ed.). Women, culture and development: a study of human capabilities. Clarendon Press, Oxford, 1995. p. 361-395.

GASPER, D. Sen's Capability Approach and Nussbaum Capabilities Ethic. Journal of International Development, USA, v. 9, n. 2, p. 281302, 1997.

GÉNERO y capacidades humanas. Granada: Comares, 2012.

GLOVER, J. The Research Programme of Development Ethics. In: NUSSBAUM, M.; GLOVER, J. (Ed.). Women, culture and development: a study of human capabilities. Clarendon Press, Oxford, 1995. p. 116-139. 
HOOKS, B. Feminist theory: from margin to center. Boston: South End Press, 1984.

IDEOLOGÍAS jurídicas y cuestión social. Los orígenes de los derechos sociales en España. Granada: Comares, 2007.

IRIGARAY, L. Espéculo de la otra mujer. Traducción R Sánchez Cedillo. Madrid: Akal, 2007.

KIERKEGAARD, S. Diary of a seducer. London: Frederik Ungar Publishing Co. Inc., 2006.

LAS FRONTERAS de la justicia. Consideraciones sobre la exclusión. Traducción A. Santos Mosquera y R. Vilá Vernis. Barcelona: Paidós, 2007.

LOCKE, J. Two treatises of goverment. Londres: Balwin, 1824.

MARX, K. Sobre la cuestión judía. Traducción comparada con la versión de la Editorial Progreso. Buenos Aires: Prometeo, 2004.

MILL, J. S. La dominación de la mujer. In: MILL, J. S. Sobre la libertad y otros escritos. Traducción J. Abellan. Madrid: M. C. C. de Iturbe, Negro Pavón, Ministerio de Trabajo y Seguridad Social, 1991. p. 148243.

MOLLER OKIN, S. Inequalities Between the Sexes in Different Cultural Contexts. In: NUSSBAUM, M.; GLOVER, J. (Ed.). Women, culture and development: a study of human capabilities. Oxford: Clarendon Press, 1995. p. 274-297.

MONEREO ATIENZA, C. Herramientas para una teoría de los derechos sociales. Anuario de Filosofía del Derecho, Valencia, v. XXII, p. $265-$ 290, 2005.

MUJERES y desarrollo humano. Traducción R. Bernet. Barcelona:

Herder S. A., 2002.

NUSSBAUM, M. Human Capabilities, Female Human Being. In: NUSSBAUM, M.; GLOVER, J. (Ed.). Women, culture and development: a study of human capabilities. Oxford: Clarendon Press, 1995. p. 61-104. 
O'NEILL, O. Justice, Capabilities and Vulnerabilities. In: NUSSBAUM, M.; GLOVER, J. (Ed.). Women, culture and development: a study of human capabilities. Oxford: Clarendon Press, 1995. p. 140-152.

PAISAJES del pensamiento. La inteligencia de las emociones. Traducción A. Maira. Cambridge: Cambridge University Press, 2008. PATEMAN, C. The sexual contract. Cambridge: Polity Press, 1988. PERFECTIONISM and Politics. Ethics, Chicago, v. 111, n. 1, p. 37-63, oct., 2000.

PULEO, A. Dialéctica de la sexualidad: género y sexo en la filosofía contemporánea. Madrid: Cátedra, 1992.

PUTNAM, H. Jewish philosophy as a guide to life: Rosenzweig, Buber, Levinas, Wittgenstein. Bloomington: Indiana University Press, 2008.

ROUSSEAU, J. J. Discurso sobre el origen y fundamentos de la desigualdad de condiciones de los hombres. Madrid: Impr. José del Collado, 1820.

SÁNCHEZ GARRIDO, P. Raíces intelectuales de Amartya Sen. Aristóteles, Adam Smith y Karl Marx. Madrid: Centro de Estudios Constitucionales, 2008.

SCHOPENHAUER, A. Eudemonología y otros pensamientos escogidos. Madrid: J. B. Bergua, Ávila, 1961.

SEN, A. Development as freedom. Nueva York: Alferd A. Knopf, 1999. TAYLOR, C. Sources of self: the making of modern identity. Cambridge: Cambridge University Press, 1989.

WOLF, S. Martha C. Nussbaum: Human Capabilities, Female Human Beings. In: NUSSBAUM, M.; GLOVER, J. (Ed.). Women, culture and development: a study of human capabilities. Oxford: Clarendon Press, 1995. p. 105-115. 
Cristina Monereo Atienza é professora titular de filosofia do Direito na Universidad de Málaga/Espanha, licenciada em Direito pela Universidad de Granada/Espanha, e doutora pela Universidad Carlos III em Madrid/Espanha. Também é licenciada em Belas Artes pela Universidad de Málaga/Espanha. Com diversos trabalhos publicados, atua na área de Filosofia do Direito em torno da igualdade e dos Direitos Fundamentais.

E-mail: cmonereo@uma.es

Endereço profissional: Facultad de Derecho, Departamento de Derecho financiero, Economía política y Filosfía del Derecho. Área de Filosofía del Derecho. Campus de Teatinos. Avenida Cervantes, 229071, Málaga. 